\title{
Partizipatorische Modernisierungstrends. Bewertung von Referenden und Lösungen zur Krise repräsentativer Demokratie am Beispiel von Stuttgart 21
}

\author{
Markus Reiners
}

\section{Kontext, Forschungsfragen und Terminologie}

Wartet eine neue Modernisierungsphase auf uns, eine Phase, bei der es um Aspekte der politischen Repräsentation und Legitimation, um Politikvermittlung oder -gestaltung geht, um Fragen, ob das repräsentative System nicht hin zu mehr direktdemokratischen Formen verschoben werden sollte? Man könnte es annehmen, beobachtet man die jüngsten Vorkommnisse um Stuttgart 21, die einen Ausstrahlungseffekt auf die gesamte Republik und darüber hinaus bewirkt und die repräsentativen Organe umfänglich beschäftigt haben, von der kommunalen bis zur europäischen Ebene. Hierbei geht es tatsächlich um mehr als um einen Bahnhof, seinen Nord- oder Südflügel, zu fällende Bäume, um Mineralquellen, die Topografie oder Erd- und Gesteinsschichten, die beim Tunnelbau Probleme aufwerfen könnten. Diese Themen sind geeignet, Unsicherheit zu erzeugen und damit Massen zu mobilisieren. Sie fungieren somit als Symboliken, die lediglich auf den eigentlichen Kern der Materie hindeuten, denn es steht sehr viel mehr auf dem Spiel. Zumindest den „Köpfen" des Protests geht es um eine Ablösung der Herrschaftsverhältnisse und um Veränderungen bei der Mitgestaltung von Politik. ${ }^{1}$

Im Zuge von Stuttgart 21 wird explizit deutlich, dass sich Bevölkerungsteile nicht mehr richtig von den etablierten Parteien vertreten fühlen. Diese scheinen immer weniger in der Lage, die sozialen Probleme politisch zu verarbeiten, was Argumente zur angeblichen Krise der Repräsentation neu belebt. ${ }^{2}$ Dies hat seinen Ursprung auch darin, dass Parteien der Rationalität des Machtzugewinns unterliegen, weshalb Fragen primär unter dem Gesichtspunkt der Wählermaximierung behandelt werden. Folglich bilden sich Formen des Politischen oder Bürgerbewegungen, die der Parteienlogik zuwiderlaufen. Direkter Parlamentarismus, partizipative Demokratie oder auch „liquid Democracy“ sind Begriffe für eine Idee, die besagt, dass eine Mixtur von repräsentativer und direkter Demokratie geeignet sein soll, das (vermeintliche) Herrschaftsproblem zu lösen. ${ }^{3}$ Eines scheint auf jeden Fall nahe zu lie-

1 Eine Ablösung der fast 60 Jahre regierenden CDU konnte am 27. März 2011 gelingen, gewiss auch bedingt durch andere Faktoren.

2 Vgl. Annette von Ohme-Reinicke, Kahlschlag als Rache, in: kontextwochenzeitung.de vom 20. Februar 2012, http://www.kontextwochenzeitung.de/debatte/46/kahlschlag-als-rache-1186.html (Abruf am 5. März 2013).

3 Vgl. Hartmut Aden (Hrsg.), Herrschaftstheorien und Herrschaftsphänomene, Wiesbaden 2004; Sebastian Jabbusch, Liquid Democracy in der Piratenpartei. Eine neue Chance für innerparteiliche Demokratie im 21. Jahrhundert?, Universität Greifswald 2011. Parallel findet eine Diskussion über die so genannte „interaktive Demokratie“ statt, mit der der Einfluss des Internets erfasst wird und wodurch die aktuellen Tendenzen befördert werden; hierzu Claus Leggewie / Christoph Bieber, Interaktive Demokratie. Politische Online-Kommunikation und digitale Politikprozesse, in: APuZ, 51. Jg. (2001), B 41/42, S. 37 - 45; Flooh Perlot, Deliberative Demokratie und Internetforen. Nur eine virtuelle Diskussion? Baden-Baden 2008. Die Meldung über Twitter, kurz 
gen: Versuchen Menschen in anderen Teilen der Welt, die „erste Stufe“ der demokratischen Leiter zu erklimmen, so schicken sich Teile unserer Gesellschaft an, die nächste Stufe zu nehmen. Sind die Deutschen ihres repräsentativen Systems überdrüssig? Ist es an der Zeit, den „nächsten Schritt“ zu tun? Oder besteht gar eine große Gefahr darin, etwas Bewährtes immer schärfer kommunikativ anzutasten? Könnte man in diesem Sinne nicht auch von „postdemokratischen Entwicklungen“ sprechen? ${ }^{4}$

Im deutschen Kontext wird immer wieder ein Demokratiedefizit konstatiert und der institutionelle Rahmen der Politik, verstanden als Dominanz des repräsentativen Musters und seiner Entscheidungsmechanismen, kritisiert. Eine verstärkte Abwendung von den dominierenden Akteuren des politischen Systems ist etwa seit den 1990er Jahren zu beobachten. ${ }^{5}$ Als Folgen ergeben sich Parteienverdrossenheit, demokratiepolitische Apathie und Erosionserscheinungen des parteipolitischen Spektrums. ${ }^{6}$ Weiterhin sind rückläufige Wahlbeteiligungen, eine kritischere Öffentlichkeit und Proteste zu diagnostizieren. Direktdemokratische Beteiligungsformen sind en vogue. Franz-Ludwig Knemeyer bemerkt: „Festzuhalten ist (...), dass Politik- und Politikerverdrossenheit nicht ihre Ursache haben in einer Zunahme politischen Desinteresses oder gar einer generellen Verweigerungshaltung (...) gegenüber der Politik, vielmehr stellen sie eine bewusste Forderung (...) nach anderen direkten - Formen politischer Beteiligung dar."7

Dabei wird immer auch debattiert, ob direktdemokratische Instrumente zur Lösung bestehender Probleme beitragen können oder neue unüberbrückbare Probleme schaffen. In

nach dem Stuttgarter „Schwarzen Donnerstag“ vom 30. September 2010, dass wieder ein Wasserwerfer vor Ort sei, bei dem es sich allerdings nicht um einen der Sicherheitskräfte, sondern um einen „karnevalistisch“von den Projektgegnern umgebauten handelte, der an den genannten Tag erinnern sollte, zog binnen weniger Minuten abermals tausende Demonstranten in den Schlossgarten. So hilfreich Neue Medien sind, so bedenklich ist der Vorgang dennoch, vergegenwärtigt man sich, dass falsche oder unvollständige Meldungen Mobilisierungseffekte in Gang setzen, die nur noch schwer steuerbar sind, und die vor allem das Gefühl erzeugen, es schneller und besser zu können als die etablierte Politik. Ein Irrglaube besteht wohl auch darin, dass das Überangebot an Medien heute ein Gefühl des „Informiertseins“ vermittelt, was zur Mitsprache herausfordert und trügerisch zu sein scheint. Vielmehr verlangt eine höhere Quantität - die sich oft umgekehrt proportional zur Qualität verhält - den kritischen Umgang mit Informationen und Selektionsqualitäten. Exakt damit scheinen viele jedoch überfordert zu sein. Gegebenenfalls ist genau das Gegenteil der Fall: Das Überangebot erzeugt einen Drift in eine Unsicherheitszone, da überkomplexe Informationen auf eine immer überkomplexere Gesellschaft treffen. Es liegt nahe zu behaupten, dass gefühlte Unsicherheiten Reaktionen hervorbringen, die auf Veränderungen zielen.

4 Vgl. Claus Leggewie, Mut statt Wut. Aufbruch in eine neue Demokratie, Hamburg 2011; Tobias Mörschel/ Christian Krell, Demokratie in Deutschland. Zustand - Herausforderungen - Perspektiven, Wiesbaden 2012; Silke I. Keil / S. Isabell Thaidigsmann (Hrsg.), Zivile Bürgergesellschaft und Demokratie, Wiesbaden 2013.

5 Vgl. Roland Roth, Die Kommune als Ort der Bürgerbeteiligung, in: Ansgar Klein / Rainer Schmalz-Bruns (Hrsg.), Politische Beteiligung und Bürgerengagement in Deutschland. Möglichkeiten und Grenzen, Baden-Baden 1997, S. 404 - 447, S. 417.

6 Vgl. Kai Arzheimer, Politikverdrossenheit. Bedeutung, Verwendung und empirische Relevanz eines politikwissenschaftlichen Begriffs, Opladen 2002; Iris Huth, Politische Verdrossenheit. Erscheinungsformen und Ursachen als Herausforderungen für das politische System und die politische Kultur der Bundesrepublik Deutschland im 21. Jahrhundert, Münster 2004.

7 Franz-Ludwig Knemeyer, Bürgerbeteiligung und Kommunalpolitik. Eine Einführung in die Mitwirkungsrechte von Bürgern auf kommunaler Ebene, München 1995, S. 51 f.; Theo Schiller I Volker Mittendorf(Hrsg.), Direkte Demokratie. Forschung und Perspektiven, Wiesbaden 2002. 
diesem Zusammenhang stellt sich die Frage, welche Bedeutung Referenden haben. Können also Formen direkter Demokratie als Instrumente der Steuerung beziehungsweise Impulsgebung und Innovation, und somit als Bereicherung des politischen Wettbewerbs verstanden werden? Gerade die Gegnerschaft zu Stuttgart 21 belegt diese Argumentationsstränge. Auf der Seite der Befürworter besteht eher die Auffassung, dass solche Wege als Instrumente der Beharrung, Verkrustung und Innovationshemmung einzuschätzen sind. ${ }^{8}$

Exakter ausgedrückt geht es um einen normativen Blick auf repräsentative und direktdemokratische Partizipationsformen, ob die systemischen Rahmenbedingungen noch hinreichend sind, oder ob es mittlerweile eine staatliche Modernisierungslücke zu verdichten gilt und wie dies geschehen könnte. Die Diskussion ist alt und zugleich aktuell wie lange nicht mehr. An dem prägnanten Beispiel Stuttgart 21 lassen sich die diesbezüglichen Vorgänge theoretisch einordnen, und es lässt sich damit erfassen, warum die Verläufe nur schwer änderbar waren. Erscheint ferner ein Umsteuern auf andere Systemvarianten nicht fruchtbar, müssen Wege gefunden werden, solche Vorhaben dennoch besser umzusetzen. Die Wissenschaft hält hierfür Antworten bereit.

Ist von direktdemokratischen Elementen die Rede, können verschiedene Instrumente unterschieden werden, die auch in ihren Wirkungen differieren ${ }^{9}$ : In der Wissenschaft werden Plebiszite als Oberbegriff für direktdemokratische Formen verwendet, unabhängig davon, ob es sich um Volksbefragungen, Volksentscheide oder Referenden handelt. Die Volksbefragung ${ }^{10}$ hat einen lediglich unverbindlichen und beratenden Charakter für den finalen Entscheid der Parlamente. Der Volksentscheid ist hingegen in aller Regel verbindlich. Er bezeichnet im überwiegenden deutschen Sprachgebrauch eine direktdemokratische Abstimmung zu einer konkreten Sachfrage des Wahlvolkes über die Annahme oder Ablehnung einer Vorlage, beispielsweise eines Gesetzes. ${ }^{11}$ In Baden-Württemberg wird der Begriff Volksabstimmung verwendet; beide Begriffe sind aber kongruent. Vorwiegend im politikwissenschaftlichen Kontext sind die beiden Termini unterschiedlich zu werten. Ist der Volksentscheid auf den förmlichen Akt einer Abstimmung gerichtet, so thematisiert die Volksabstimmung umfassend den mehrstufigen Akt der Gesetzgebung, also die Volksinitiativen, das Volksbegehren und den -entscheid. In der Politikwissenschaft wird der Begriff des Volksentscheides stets verwendet, um die Abstimmung über eine vom Volk vorgebrachte Vorlage zu einem politischen Gegenstand (so genanntes Initiativverfahren) zu bezeichnen. Ein Referendum ist im Gegensatz hierzu stets definiert als eine Abstimmung über eine von der gewählten Volksvertretung (Parlament) oder der Regierung erarbeitete Vorlage. ${ }^{12}$

8 Vgl. Wolfgang Luthardt, Direkte Demokratie. Ein Vergleich in Westeuropa, Baden-Baden 1994, S. 23 ff.; Mario Martini, Wenn das Volk (mit)entscheidet. Wechselbeziehungen und Konfliktlinien zwischen direkter und indirekter Demokratie in der Rechtsordnung, Berlin 2011; Eike-Christian Hornig, Direkte Demokratie und Parteienwettbewerb. Überlegungen zu einem obligatorischen Referendum als Blockadelöser auf Bundesebene, in: ZParl, 42. Jg. (2011), H. 3, S. 475 - 492.

9 Vgl. Klaus Schubert / Martina Klein, Das Politiklexikon, Bonn 2011; Otmar Jung, Grundgesetz und Volksentscheid. Gründe und Reichweite der Entscheidungen des Parlamentarischen Rats gegen Formen direkter Demokratie, Opladen 1994.

10 Der Ausdruck Volksbefragung wird ausschließlich im Grundgesetz verwendet und findet sich in keiner Landesverfassung; vgl. Art. 29 GG.

11 Im Unterschied hierzu wird bei der Wahl über die Vergabe von Mandaten an Personen entschieden; vgl. Art. 20 GG.

12 Diese Unterscheidung findet sich nicht in den deutschen Verfassungen und Gesetzen. So spricht das GG durchweg von Volksentscheiden. Auch die Landesverfassung von Baden-Württemberg 


\section{Direkter vs. repräsentativer Mechanismus: Dialektische Argumentationsstränge und differierende Topoi}

In sämtlichen Bundesländern sind Referenden inzwischen installiert, auf der obersten Ebene ist das Grundgesetz jedoch - mit einer kleinen Ausnahme - auch nach den Verfassungsergänzungen im Hinblick auf Volksabstimmungen hermetisch abstinent geblieben. ${ }^{13}$ Dabei gilt es zu bedenken, dass ein Vergleich von Baden-Württemberg mit der Bundesebene manchen Betrachtern näher kommt als mit den meisten anderen Ländern, weil bislang das Abstimmungsquorum für direkte Beteiligungsformen im Südwesten vergleichsweise hoch angesetzt ist (33 Prozent bei einfachen Gesetzen) - was die Frage erlaubt, ob das Instrument damit nicht zu einer theoretischen Größe verkommt, wobei aktuell wieder über eine Absenkung des Quorums diskutiert wird, ohne dass bislang Ergebnisse vorliegen, weil hierzu die Landesverfassung geändert werden muss. ${ }^{14}$

Ein Argument zur wirkungsmächtigeren Ausweitung von Beteiligungsmöglichkeiten lautet, Demokratie könne sich nicht auf Wahlen beschränken und in der übrigen Zeit der Bürgerschaft nur eine Zuschauerrolle überlassen. Sie sei dem Wesen nach darauf gerichtet, so das rousseauistische Demokratieverständnis, die Identität von Regierenden und Regierten herzustellen. Ein Staat könne daher nur dann als demokratisch bezeichnet werden, wenn er seine Bürger umfassend an der Willensbildung beteiligt und Referenden zwischen den Wahlen installiert damit eine höhere Legitimität entsteht. ${ }^{15}$ Demgegenüber wird allerdings behauptet, dass in repräsentativen Systemen genügend Einflussformen bestehen. ${ }^{16}$ Wichtiger noch: Rousseaus Vorstellung, mit der Identität von Regierenden und Regierten werde Herrschaftsfreiheit erreicht, wird die repräsentative Demokratie entgegengesetzt und theoretisch sogar als die „eigentliche" Form der Demokratie begründet. ${ }^{17}$

differenziert nicht, ob bei einer direktdemokratischen Abstimmung über eine Vorlage des Volkes oder über eine Vorlage des Parlaments respektive der Regierung entschieden wird. Hier wird im weiteren Fortgang vielfach der Begriff eines Referendums verwendet.

13 Vgl. Wolfgang Luthardt / Arno Waschkuhn, Plebiszitäre Komponenten in der repräsentativen Demokratie. Entwicklungsstand und Perspektiven, in: Ansgar Klein / Rainer Schmalz-Bruns (Hrsg.), a.a.O. (Fn. 5), S. $59-87$.

14 Vgl. Verfassung des Landes Baden-Württemberg vom 11. November 1953 (GBl. S. 173), zuletzt geändert am 7. Februar 2011 (GBL S. 46), Art. 60 Abs. 5. Arnold Rieger / Frank Krause, Neues Quorum 33 oder 25 oder 20 oder gar nichts?, in: Stuttgarter Nachrichten am 28. November 2011, http://www.stuttgarter-nachrichten.de/inhalt.neues-quorum-33-oder-25-oder-20-oder-garnichts.c697157b-07c2-4b58-954a-e733a7687be8.html; Nadine Michel, Direkte Demokratie im Ländle. 20 Prozent reichen, in: taz am 7. November 2013 (jeweils Abruf am 11. September 2014).

15 Vgl. Frank Hollihn, Partizipation und Demokratie. Bürgerbeteiligung am kommunalen Planungsprozess?, Baden-Baden 1978, S. 9 ff., S. 79.

16 Vgl. Ansgar Klein / Rainer Schmalz-Bruns, Herausforderungen der Demokratie. Möglichkeiten und Grenzen der Demokratisierung, in: dies. (Hrsg.), a.a.O. (Fn. 5), S. 7 - 38, S. 19; Bernhard Kornelius / Dieter Roth, Politische Partizipation in Deutschland. Ergebnisse einer repräsentativen Umfrage, Bonn 2004; Rudolf Wassermann, Die Zuschauerdemokratie, Düsseldorf 1996; Winfried Steffani, Pluralistische Demokratie, Opladen 1980, S. $151 \mathrm{ff}$.

17 Vgl. Ernst-Wolfgang Böckenförde, Mittelbare/repräsentative Demokratie als eigentliche Form der Demokratie. Bemerkungen zu Begriff und Verwirklichungsproblemen der Demokratie als Staatsund Regierungsform, in: Georg Müller (Hrsg.), Staatsorganisation und Staatsfunktionen im Wandel. Festschrift für Kurt Eichenberger zum 60. Geburtstag, Basel / Frankfurt am Main 1982, S. $301-328$, S. 314. 
Nach weiteren damit verknüpften Funktionszuschreibungen dienen Referenden als Informations- und Artikulationsquelle für die Willensbildung der Bürger und Entscheider. Zugleich böten sie die Chance, als Mechanismus einer Sozialintegration zu wirken. Im Hinblick auf politische Themen könne ein Protestpotential in diverse Dimensionen und Stufen des Prozesses einbezogen werden. Ferner wird ihr Bezug auf die Öffentlichkeit sowie die Politikintegration betont, gemeint ist die Kontroll-, Balance- und Vetofunktion. ${ }^{18}$ Referenden werden als „staatsrechtliches Gegengewicht“ interpretiert und als „Mittel demokratischer Balancierung und Kontrolle der Parteienmacht " rezipiert. ${ }^{19}$ Anderer Natur ist ein Argument mit entscheidungstheoretischem Hintergrund, das von zunehmender Komplexität ausgeht. Die immer größere Datenflut zwinge zu immer besseren und rationelleren Methoden der Informationsverarbeitung und -beschaffung. Sinnvoll und zielgerichtet könne nur geplant werden, wenn die Bedürfnisse der Betroffenen bekannt seien. ${ }^{20}$

Befürworter referendumsdemokratischer Prozesse erhoffen sich einen in ihrem Interesse liegenden Transfer ihrer Vorstellungen in die Politikarenen. Normativ orientierte Thesen reklamieren, dass Formen direkter Demokratie einen Beitrag zur Differenzierung des institutionellen Gefüges liefern können. Hierbei wird nicht nur eine intensivere Partizipation, sondern überdies ein besseres Politikergebnis im Vergleich zu den repräsentativen Politikentscheidungen angenommen. ${ }^{21}$ Dem wird entgegengehalten, dass Partizipation sicherlich nicht von vornherein ein besseres Ergebnis hervorbringt als Nicht-Partizipation. Maßgeblich kommt es auf deren Qualität an, die letztlich daran gemessen werden muss, was sie zum Ziel, eine sachlich angemessene und demokratisch vertretbare Entscheidung zu fällen, tatsächlich beizutragen vermag. Allerdings macht ein höheres Maß an Partizipation den Willensbildungs- und Entscheidungsprozess unter Umständen komplizierter. Daraus folgt, dass eine sinnvolle Bürgerbeteiligung, auch wenn man in ihr mit guten Gründen ein positives Element der Demokratie sehen mag, nicht ohne ein Minimum an Regeln auskommt. Diese dürfen aber nicht so kompliziert sein, dass dadurch ein sachlich optimales Ergebnis unmöglich wird. ${ }^{22}$

Wolfgang Luthardt bezieht eine maximale Position und diagnostiziert, dass es nicht möglich sei, eine höhere Legitimation und ein besseres Politikergebnis im Vergleich zu repräsentativen Entscheidungen zu erzielen. Aus seiner Sicht rufen Formen direkter Demokratie Politikblockaden und entscheidungspolitische Verzögerungen hervor. Der Produktivfaktor Partizipation würde sich somit restriktiv auswirken. ${ }^{23}$ Skeptiker befürchten gerade, dass

18 Vgl. Wolfgang Luthardt, a.a.O. (Fn. 8), S. $158 \mathrm{f}$.

19 Ernst-Wolfgang Böckenförde, Staat, Verfassung, Demokratie, Frankfurt am Main 1991, S. 371.

20 Vgl. Frank Hollihn, a.a.O. (Fn. 15), S. 9 ff.

21 Vgl. Rudolf Wassermann, a.a.O. (Fn. 16). Die Argumentation verläuft dergestalt, dass es durch die Erweiterung des institutionellen Rahmens um Plebiszite möglich wird, institutionelle Hürden zu überspringen, Themen in legislative Arenen zu transportieren und erhoffte Ergebnisse zu erzielen. Instrumente direkter Demokratie erscheinen so als partizipations-, steuerungs- und themenorientierte Instrumente des Entscheidungsprozesses. Eine strukturelle Erweiterung des Inputs führe zu funktionell positiven Resultaten beim Output. Referenden wären in der Lage, Blockaden aufzulösen, was als Ergebnis ihrer optimierenden Integrations- und Korrekturfunktion gedeutet wird.

22 Vgl. Kurt Sontheimer, Bürgerbeteiligung in der repräsentativen Demokratie, in: Uwe J. Walther I Astrid Zellmer (Hrsg.), Bürgerbeteiligung, Bürgermitwirkung, Bürgerselbsthilfe. Dokumentation von Vorträgen einer gemeinsamen Veranstaltung des Instituts für Städtebau Berlin und der Bundesforschungsanstalt für Landeskunde und Raumordnung von 25. bis 27. Januar 1988, Bonn 1988, S. $1-8$, S. 6.

23 Vgl. Wolfgang Luthardt, a.a.O. (Fn. 8), S. 26, S. 164 f. 
direkte Beteiligungsformen zu Erosionsprozessen der repräsentativen Politikkontexte führen. Die institutionelle Demokratietheorie fügt der Debatte einen weiteren Aspekt hinzu: Die Aufgabenbewältigung hänge hauptsächlich von der Form der Demokratie ab. Eine Referendumsdemokratie, wie die Schweiz, wäre für Begehren besser gerüstet als ein Staat mit dominierender Repräsentativverfassung. In reinen repräsentativen Demokratien führe die partizipatorische Revolution zwingend zu einer Zunahme unverfasster politischer Beteiligung und zu einer höheren Wahrscheinlichkeit von Konflikten. Allerdings wird eine größere Responsivität, so zeige es sich in der Schweiz, mit hohen Entscheidungs- und Konsensbildungskosten sowie einer größeren Wahrscheinlichkeit von Prozessblockaden erkauft. ${ }^{24}$

Ein zentrales Argument lautet, dass direktdemokratische Entscheidungen die Politikkomplexität nicht adäquat verarbeiten können und damit einer Steuerungsfähigkeit zuwiderlaufen. Es wird unterstellt, dass die Logik referendumsdemokratischer Entscheidungen auf eine Negation von Kompromissen hinauslaufe. Diese stünden quer zu den differenzierten und kompromisshaft vorstrukturierten Entscheidungen repräsentativer Institutionen. ${ }^{25}$ „Die Überprüfung der Beschlüsse der Parlamente durch das ,Referendum` bedeutet (...) eine wesentliche Stärkung aller irrationalen Mächte des Beharrens, weil sie Feilschen und Kompromiss zwischen den Interessenten (...) ausschließt." 26

Die Formel „mehr direkte Demokratie = mehr Volkssouveränität“ ist insofern plakativ und verführerisch, aber gegebenenfalls zu einfach. Erklärt wird, dass sie als ein vermeintlicher Königsweg der Demokratie und der damit verbundenen Problemkomplexität keineswegs angemessen und - als ein gleichsam auf den Kopf gestelltes Nullsummenspiel - zu undifferenziert sei. Die emphatische Steigerungsformel wäre theoriearm und praxisfern, weil sie nicht nur unterkomplex sei, sondern darüber hinaus Konflikte oder Machtverlagerungen unterschätze. Alle generalisierbaren Erfahrungen in den sich deutlich abschwächenden Proporz- oder Konkordanzdemokratien zeigen, vor allem in der Schweiz, dass mit plebiszitären Volksrechten stets auch retardierende und innovationshemmende Momente einhergehen und Prozesse bis an den Rand des Unvertretbaren verlängert werden. ${ }^{27}$

Ein weiterer Aspekt wird in der fehlenden Responsivität direktdemokratischer Entscheidungen gesehen. Diese verfügen über keine identifizierbare Person, Partei oder Institution, die für das Ergebnis Verantwortung übernimmt. ${ }^{28}$ In theoretischer Hinsicht kommt bei ihnen ein reduziertes Entscheidungsverhalten zum Vorschein. Dieses verläuft nach einer einfachen Ja/Nein-Logik. ${ }^{29}$ Es fehle eine reflexive Rückbindung, ein „reflexive loop“.30

24 Vgl. Manfred G. Schmidt, Komplexität und Demokratie. Ergebnisse älterer und neuerer Debatten, in: Ansgar Klein / Rainer Schmalz-Bruns (Hrsg.), a.a.O. (Fn. 5), S. 41 - 58, S. 42 ff.

25 Vgl. Wolfgang Luthardt, a.a.O. (Fn. 8), S. 159 f.

26 Max Weber, Wirtschaft und Gesellschaft, Tübingen 1972, S. 767.

27 Vgl. Wolfgang Luthardt / Arno Waschkuhn, a.a.O. (Fn. 13), S. 60; Silvano Möckli, Direkte Demokratie: ein Vergleich der Einrichtungen und Verfahren in der Schweiz und Kalifornien, unter Berücksichtigung von Frankreich, Italien, Dänemark, Irland, Österreich, Liechtenstein und Australien, Bern 1994.

28 Vgl. Wolfgang Luthardt, a.a.O. (Fn. 8), S. 60; Ernst-Wolfgang Böckenförde, a.a.O. (Fn. 19), S. 396.

29 Vgl. Norberto Bobbio, The Future of Democracy, Cambridge 1987, S. 117; Wolfgang Luthardt, a.a.O. (Fn. 8), S. 160.

30 Claus Offe, Disorganized Capitalism, Oxford 1985, S. 296. 
Ja/Nein-Entscheidungen „do not include the opportunities of a critical evaluation of multiple individual preferences based on argumentation and discourse" ${ }^{31}$. Fraglich ist daher umso mehr, ob Demokratie mit zunehmender Partizipation, wachsender Komplexität und einer Begrenzung politischer Steuerung zurechtkommt oder hiervon nicht überfordert wird. Die Familien der Demokratietheorie beantworten diese Frage unterschiedlich, auf einem Kontinuum von positiven bis zu eher pessimistischen Schlussfolgerungen.

Aus politiktheoretischer Sicht erscheinen die Argumente durchaus ausgewogen zu sein. Wolfgang Luthardt und Arno Waschkuhn sehen die Topoi in demokratie-, legitimations- und partizipationstheoretische Vorstellungen eingebettet, die sich vor allem auf Fragen der Relation von politischen Eliten zu Nicht-Eliten, auf Repräsentation, auf die mögliche Entoder Umwertung des Parlaments, den Stellenwert der politischen Führung und das Ausmaß politischer Beteiligung in einer Massendemokratie beziehen, die eine Versammlungsdemokratie ausschließt. In zugespitzter Weise geht es, so die Autoren, um die Frage einer Basis- (bottom up) oder Elitendemokratie (top down) als Strukturmuster soziopolitischer Vermittlungsprozesse. Sie führen stichhaltig die wesentlichsten Pro- und Contrapunkte einer Referendumsdemokratie an. ${ }^{32}$

Auf der einen Seite wird die Stärkung der Volkssouveränität, die Verwirklichung des Demokratiepostulats, die Akzentuierung des Wertberücksichtigungs- beziehungsweise Konfliktpotentials und das Gegenprinzip zur Zuschauerdemokratie hervorgehoben. Direkte Demokratie schöpft gegebenenfalls das Humanpotential sowie das soziale Kapital des Gemeinwesens individuell und kollektiv besser aus. Solche Verfahren sorgen generell für Transparenz und lösen grundlegende Kontroversen beziehungsweise führen zu einer hohen Akzeptanz und Legitimation. Außerdem fördert direkte Demokratie die politische Sozialisation und Erziehung zur Mündigkeit, baut Entfremdungsphänomene ab und setzt widerständige Triebkräfte gegen Anmaßungen der politischen Klasse in produktiver Weise frei. $^{33}$

Auf der anderen Seite wird erklärt, dass direkte Demokratie wohl nur zu überschaubaren territorialen Einheiten passt. Die Komplexität in fortgeschrittenen Industriegesellschaften schließt breit angelegte direktdemokratische Verfahren aus, da der Citoyen hiervon unter Umständen überfordert ist und im Schnitt über geringere Sachkompetenzen verfügt. Eine permanente Beschäftigung mit öffentlichen Angelegenheiten ist daher nicht von allen zu erwarten. Vielmehr bedarf es einer sachverständigen politischen Führung. Zufallskonstellationen und Stimmungsschwankungen geben bei direktdemokratischen Verfahren den Ausschlag und implizieren insofern keine Entscheidungsrationalität. Eine Reduzierung auf ein Antwortverhalten nach dem Muster „Alles oder Nichts“ ist überdies zu simpel und bietet keinen Raum für Zwischenlösungen und Kompromisse. Das Volk kann ferner immer nur punktuell entscheiden, und vielfach artikulieren sich lediglich engagierte Minderheiten. Letztendlich dauern solche Verfahren lange, behindern oft Innovationen und befördern eine Tyrannei der Mehrheit. Es ist daher zweifelhaft, ob die Gesetzesqualität verbessert wird

31 Adrienne Windhoff-Héritier, Institutions, Interests and Political Choice, in: Roland Czada / dies. (Hrsg.), Political Choice. Institutions, Rules and the Limits of Rationality, Frankfurt am Main 1991, S. 27 - 52, S. 44.

32 Vgl. Wolfgang Luthardt / Arno Waschkuhn, a.a.O. (Fn. 13), S. 60 f.

33 Vgl. ebenda. 
sowie Konflikte entschärft werden und sich nicht partikulare Anliegen durchsetzen, wenn sich nur wenige am Entscheid beteiligen und in geheimen Abstimmungen votieren. ${ }^{34}$

Skepsis, bestenfalls Ambivalenz, ist nach den bisherigen Ausführungen angebracht, wenn es um die Perspektiven formeller Bürgerbeteiligung geht. ${ }^{35}$ Schon Kurt Sontheimer führte aus, dass, bevor das Verlangen nach mehr Partizipation stärker artikuliert und diskutiert wurde, man sich mit der vom Grundgesetz gewollten repräsentativen Struktur abgefunden hatte. Die Mitwirkung des Volkes an Wahlen wurde für eine unerlässliche, aber auch ausreichende Form gehalten. ${ }^{36}$ Der Wahlakt selbst ist der entscheidende Akt der Legitimation; die repräsentative Demokratie - deren Ablösung nicht zur Debatte steht - bedarf jedoch unter Umständen der Abstützung durch weitere Elemente, denn schon seit längerer Zeit wächst Unbehagen an der repräsentativen Struktur. ${ }^{37}$

Die Argumente sprechen für und gegen einen wirkungsvolleren Ausbau direktdemokratischer Instrumente. Auch wenn vielfach behauptet wird, dass eine direkte Demokratie hierzulande nicht funktionieren kann, dürfte unumstritten sein, dass die Möglichkeiten, die durch das Grundgesetz geschaffen wurden, an Grenzen angelangt sind, und die Forderungen nach mehr direkter Beteiligung ihre Berechtigung haben. Auch die repräsentative Variante funktioniert nicht vollständig, weil die globalisierte Welt einem schnelllebigen Wandel unterliegt und sich politische Einstellungen schneller ändern als noch im 20. Jahrhundert. Fraglich ist somit mehr denn je: Kommt es wirklich auf Referenden an? Klar ist, dass eindimensionale Erklärungen ausscheiden. Nach dialektisch abwägendem Blick lautet ein normatives Zwischenfazit: Als Ergebnis vermehrter direkter formeller Partizipation kristallisiert sich eine höhere legitimatorische Abstützung von Entscheidungen auf Kosten der Steuerungs- und Problemlösungsfähigkeit heraus. Bestehende Probleme werden durch mehr direkte Demokratie wohl nicht erheblich reduziert. Vielmehr scheint es so, dass neue Probleme hinzutreten und Innovationen erschwert werden.

Diskussionen über das politische System sind in dieser diametralen Maximalität somit wenig ertragreich. Dass dennoch eine Modernisierungslücke besteht, ist offenbar. Dies zeigt sich überdeutlich am Beispiel Stuttgart 21. Ist also ein Umsteuern auf grundlegend andere systemische Voraussetzungen nicht fruchtbar, so sollte keinesfalls die Frage verworfen werden, wie solche Vorhaben künftig besser lösbar sind.

\section{Stuttgart 21: Meilensteine, Restriktionen und theoretische Lösungswege}

\subsection{Verlauf des Großprojekts}

Bereits im Jahr 1994 wurde das Projekt Stuttgart 21 der Öffentlichkeit vorgestellt, das unter anderem einen unterirdischen Bahnhof vorsieht. Ende 1995 verständigten sich Land, Stadt und Region mit der Deutschen Bahn AG und dem Bund grundsätzlich auf das Vor-

34 Vgl. ebenda; Herbert Kitschelt, Demokratietheorie und die Veränderungen politischer Beteiligungsformen. Zum institutionellen Design postindustrieller Gesellschaften, in: Forschungsjournal Neue soziale Bewegungen, 9. Jg. (1996), H. 2, S. 61 - 69; Roland Roth, a.a.O. (Fn. 5), S. 440.

35 Vgl. Roland Roth, a.a.O. (Fn. 5), S. 441.

36 Vgl. Kurt Sontheimer, a.a.O. (Fn. 22).

37 Vgl. Bettina Westle, Politische Partizipation, in: Oskar W. Gabriel / Frank Brettschneider (Hrsg.), Die EU-Staaten im Vergleich. Strukturen, Prozesse, Politikinhalte, Opladen 1994, S. 137 - 173. 
haben. In der Folgezeit wurden die Finanzierungsmodalitäten sondiert. 2001 liefen die Planfeststellungsverfahren an, der Planfeststellungsbeschluss erfolgte im Februar 2005. Im April 2003 begann ein Erörterungsverfahren, bei dem das Regierungspräsidium Stuttgart über 5.000 private Einwendungen prüfte. Im April 2006 wies der VGH Baden-Württemberg mehrere Klagen von Projektgegnern zurück. ${ }^{38}$

Im Juni 2006 vereinbarten die Partner sodann, über das Projekt und die noch nicht abschließend geklärte Finanzierung zu entscheiden. Ein Hauptstreitpunkt waren hierbei die Kostenschätzungen. So mussten die Prognosen seit den 1990er Jahren mehrfach von rund 2,5 Milliarden Euro bis auf über vier Milliarden Euro angehoben werden. Eine Schätzung vom Dezember 2009, auf Basis der Entwurfsplanung und mit Preisstand von 2009, bezifferte die Kosten in etwa auf die vorgenannte Summe. ${ }^{39}$ Die Bahn trägt hiervon den Löwenanteil neben dem Bund, der mit EU-Mitteln beteiligt ist. Das Land übernimmt einen geringeren Anteil, und deutlich kleinere Beträge entfallen auf die Stadt, den Flughafen und die Region Stuttgart. Im März 2012 betrug der Gesamtumfang des Projektes nach Aussagen der Deutschen Bahn bereits über 4,3 Milliarden Euro und im Dezember 2012 knapp 4,7 Milliarden Euro. ${ }^{40}$ Darüber hinaus wurden zusätzliche Risiken aus externen Einflussfaktoren in Höhe von knapp einer Milliarde Euro genannt. Angesichts der Mehrkosten schlug die Deutsche Bahn vor, den Rahmen der Finanzierung mit Eigenmitteln auf über 5,6 Milliarden Euro aufzustocken. Zwischen diesem Betrag und den geschätzten Kosten würde sich demnach ein Puffer von über 900 Millionen Euro ergeben.

Blickt man auf die Beschlüsse des Landes, so ist festzustellen, dass der Landtag das Projekt im Oktober 2006 mit 115 gegen 15 Stimmen aus der Fraktion der Grünen beschlossen hat. ${ }^{41} \mathrm{Im}$ Juli 2007 einigten sich die Projektpartner über die Kostenaufteilung. ${ }^{42}$ Im April 2009 wurde schließlich die Finanzierungsvereinbarung unterzeichnet. ${ }^{43}$ Am 16. Dezember 2009 empfahl der Verkehrsausschuss des Bundestages, einen Antrag der Landtagsfraktion der Grünen abzulehnen. Darin war die Bundesregierung aufgefordert worden, ein Moratorium für das Projekt zu verhängen. Am Folgetag wurde der Antrag im Plenum abgelehnt. ${ }^{44}$

38 Vgl. Stephan Orth / Antje Blinda, „Stuttgart 21“: Stationen eines umstrittenen Mega-Bauprojekts, in: Spiegel online vom 19. Juli 2007, http://www.spiegel.de/reise/aktuell/stuttgart-21-stationeneines-umstrittenen-mega-bauprojekts-a-495408.html (Abruf am 5. März 2013).

39 Vgl. Landtag von Baden-Württemberg, LT-Drs. 14/4382 vom 22. April 2009; Nikolaus Doll I Frank Seidlitz, Bahn feuert Vorstand nach „Stuttgart 21“-Debakel, in: Welt online vom 10. Dezember 2009, http://www.welt.de/welt_print/wirtschaft/article5483083/Bahn-feuert-Vorstandnach-Stuttgart-21-Debakel.html (Abruf am 12. Dezember 2012).

40 Vgl. Volker Kefer, Stuttgart 21 und NBS Wendlingen-Ulm. Unterlage für den Prüfungs- und Complianceausschuss (PCA) der DB und den Lenkungskreis (LK) S21, Berlin / Stuttgart 2012, S. 8, S. 21.

41 Vgl. Landtag von Baden-Württemberg, PIPr. 14/10 vom 12. Oktober 2006.

42 Vgl. Stephan Orth / Florian Harms, Milliardenprojekt: Finanzierung für Stuttgart 21 steht, in: Spiegel online vom 19. Juli 2007, http://www.spiegel.de/reise/aktuell/milliardenprojekt-finanzierung-fuer-stuttgart-21-steht-a-495398.html (Abruf am 19. Januar 2012).

43 Vgl. Stephan Orth, Minister besiegeln Milliardenprojekt der Bahn, in: Spiegel vom 2. April 2009, http://www.spiegel.de/reise/aktuell/stuttgart-21-minister-besiegeln-milliardenprojekt-der-bahna-617037.html (Abruf am 5. März 2013).

44 Vgl. Deutscher Bundestag, BT-Drs. 17/268 vom 16. Dezember 2009 zum Antrag von Abgeordneten der Fraktion Bündnis 90/Die Grünen, BT-Drs. 17/125. 
Im November 2007 wurden im Stuttgarter Rathaus über 60.000 Unterschriften für ein Bürgerbegehren übergeben, das auf den Projektausstieg der Stadt abzielte - notwendig waren 20.000. Der Antrag auf Zulassung des Bürgerentscheids wurde im Dezember 2007 vom Stuttgarter Gemeinderat mit 45 zu 15 Stimmen mit der Begründung abgelehnt, dass dieser rechtlich unzulässig sei. Das Regierungspräsidium Stuttgart und das VG Stuttgart wiesen die Widersprüche zurück. Seit der Entscheidung für die Umsetzung gab es viele Protestaktionen, und seit November 2009 finden bis heute wöchentlich so genannte Montagsdemonstrationen mit zahlreichen Teilnehmern statt. ${ }^{45}$ Am 30. September 2010 kulminierte der Protest gegen die vorbereitenden Maßnahmen im Schlossgarten. Etwa 400 Menschen wurden dabei verletzt. ${ }^{46}$

Um die Fronten aufzubrechen, fanden vom 22. Oktober bis 27. November 2010 acht von Heiner Geißler moderierte Schlichtungsgespräche zwischen Vertretern von Projektbefürwortern und -gegnern statt. ${ }^{47}$ Am 30. November 2010 sprach er sich in seinem Schlichterspruch im Grundsatz für das Projekt aus, schlug jedoch eine Reihe von Veränderungen vor („S 21 Plus“). Welche Vorschläge zur Kapazitätssteigerung zu realisieren wären, sollte nach einem „Stresstest“ entschieden werden, dessen Ergebnisse im Juli 2011 vorgestellt wurden. Demnach könnte der Durchgangsbahnhof 30 Prozent mehr Zugankünfte zur Spitzenstunde verarbeiten. ${ }^{48}$

Im Koalitionsvertrag vereinbarten Grüne und SPD, nach dem angestrebten Regierungswechsel vom März 2011 eine Volksabstimmung (Referendum) durchzuführen. ${ }^{49}$ Ende Juli 2011 wurde hierzu ein Entwurf für ein S21-Kündigungsgesetz vorgelegt. ${ }^{50}$ Das Gesetz wurde am 29. September 2011 im Landtag abgelehnt. Damit war der Weg für ein Referendum frei, das am 27. November 2011 stattfand. Zur Abstimmung stand, ob das Land Kündigungsmöglichkeiten aus dem Finanzierungsvertrag nutzen und so aus dem Projekt aussteigen solle. ${ }^{51}$ 58,8 Prozent der Abstimmenden sprachen sich gegen die Verpflichtung der Regierung aus, Kündigungsrechte zur Aufösung der vertraglichen Vereinbarungen mit Finanzierungspflichten des Landes auszuüben, 41,2 Prozent waren dafür. Die Beteiligung

45 Vgl. Johannes Schweikle, Vereint gegen das Milliardenloch, in: Zeit online vom 11. März 2010, http://www.zeit.de/2010/11/WOS-Stuttgart (Abruf am 12. Dezember 2012).

46 Vgl. „Stuttgart 21. Hunderte Verletzte nach Polizeieinsatz“, in: Focus online vom 30. September 2010, http://www.focus.de/politik/deutschland/stuttgart-21-hunderte-verletzte-nach-polizeieinsatz_aid_557820.html (Abruf am 18. Dezember 2012).

47 Vgl. Gabriele Renz, Faktencheck im Rathaus, in: FR online vom 22. Oktober 2010, http://www. fr-online.de/politik/schlichtung-zu-stuttgart-21-beginnt-faktencheck-im-rathaus, 1472596, 4764162.html (Abruf am 7. Januar 2013).

48 Vgl. „Geißler hat eine große Chance verpasst“, in: faz.net vom 1. Dezember 2010, http://faz-archiv-approved.faz.net/intranet/biblionet/r_suche/webcgi?START =A20\&DOKM=65353_FAZN _0\&WID=89155-4530214-92830_3 (Abruf am 7. Januar 2013).

49 Vgl. Bündnis 90/Die Grünen / SPD (Hrsg.), Der Wechsel beginnt. Koalitionsvertrag zwischen Bündnis 90/Die Grünen und der SPD Baden-Württemberg 2011-2016, S. 30 f.

$50 \mathrm{Vgl}$. Staatsministerium Baden-Württemberg (Hrsg.), Landesregierung gibt Entwurf eines S 21-Kündigungsgesetzes zur Anhörung frei, 26. Juli 2011, http://www.stm.bwl.de (Abruf am 12. August 2012).

51 Vgl. Staatsministerium Baden-Württemberg, S21-Volksabstimmung ist neues Kapitel der badenwürttembergischen Demokratie, http://www.stm.bwl.de (Abruf am 12. August 2012). 
lag bei 48,3 Prozent. ${ }^{52}$ Nach Art. 60 Abs. 5 Satz 2 der Landesverfassung bedarf es zur Annahme eines Gesetzentwurfs durch das Volk nicht nur der Mehrheit der abgegebenen gültigen Stimmen, sondern darüber hinaus der Zustimmung von mindestens einem Drittel aller Stimmberechtigten. ${ }^{53}$ Damit verbunden ist eine heikle „demokratietheoretische Frage“, die sich aus den drei folgenden Abstimmungskonstellationen ergibt.

Eingetreten ist der zur Befriedung tendenziell günstige Fall, dass sich eine Mehrheit für das Projekt aussprach. Wäre allerdings die Variante eingetreten, dass die S21-Gegner in der Mehrzahl gewesen wären und das Quorum erreicht hätten, hätte dies sicherlich nicht zwangsläufig das Ende von Stuttgart 21 bedeutet, denn es war nicht über das Projekt als Ganzes, sondern lediglich über den Finanzierungsanteil des Landes zu entscheiden. Hätte sich das Land in diesem Fall von dem Projekt verabschiedet, wären enorme Schadensersatzzahlungen fällig geworden. Die Projektpartner hätten zwar rechtlich die Möglichkeit gehabt, am Vorhaben festzuhalten, es allerdings gegen den mit der konkreten Finanzierungsfrage mitschwingenden Willen des Volkes „gegen das Projekt“ politisch durchzusetzen, wäre eine höchst brisante Angelegenheit gewesen, die weitere heftigste Protestaktionen hervorgerufen hätte. Die schwierigste Konstellation wäre eingetreten, wenn die Gegner die Mehrheit erreicht und hierbei das Quorum verfehlt hätten; spätestens dann wäre es wirklich nicht mehr um den Bahnhof gegangen, sondern um Fragen mangelnder direkter Partizipationsmöglichkeiten auf der Landesebene. Prinzipielle Aspekte wären daher sicherlich noch mehr ins Zentrum der Debatte gerückt.

\subsection{Neoinstitutionalistische Hemmnisse}

Seit der Volksabstimmung ist das Projekt noch stärker institutionell abgesichert. Aber auch schon zuvor wäre es nur schwer zu stoppen gewesen. Allenfalls die zweite Variante wäre eine zumindest theoretische Option gewesen; alle anderen denkbaren Möglichkeiten stützen diese These. Hierfür liefern diverse Ansätze genügend Hinweise, besonders neoinstitutionalistische Konzepte wie der historische und der akteurzentrierte Institutionalismus. ${ }^{54}$

Diese betonen, dass Institutionen Präferenzen formen, und diese keinesfalls exogen vorgegeben sind. Es ist das Umfeld, das die Ziele und die Wahl der Mittel prägt, allerdings ohne Determination, denn zwischen Institution und Handlung wirkt die Wahrnehmung und Interpretation der Akteure. Der historische Institutionalismus betont die von Institutionen ausgehenden Pfadabhängigkeiten. Damit lässt sich erklären, dass die Präferenzen und Wahlhandlungen der Akteure durch langfristig angelegte institutionelle Kanäle vorstruktu-

52 Vgl. Statistisches Landesamt Baden-Württemberg, Vorläufiges Ergebnis der Volksabstimmung am 27. November 2011 über die Gesetzesvorlage „Gesetz über die Ausübung von Kündigungsrechten bei den vertraglichen Vereinbarungen für das Bahnprojekt Stuttgart 21 (S 21-Kündigungsgesetz)“, http://www.statistik.baden-wuerttemberg.de (Abruf am 28. November 2011); Volker Haug, Volksgesetzgebung auf verfassungsrechtlichem Neuland. Rechtsfragen im Zusammenhang mit der baden-württembergischen Volksabstimmung über das „S 21-Kündigungsgesetz“, in: ZParl, 43. Jg. (2012), H. 2, S. $446-466$.

53 Vgl. Verfassung des Landes Baden-Württemberg, a.a.O. (Fn. 14).

54 Vgl. Markus Reiners, Reform der Mittelbehörden. Rahmenbedingungen und Parameter zur Durchsetzung einer radikalen Strukturreform, in: dms, 1. Jg. (2008), H. 2, S. $303-324$, S. $319 \mathrm{ff}$. 
riert sind. Sie bewegen sich innerhalb eines Pfades, dessen Spielräume durch die in der Vergangenheit ausgebildeten Strukturen und eingeübten Muster bestimmt sind. ${ }^{55}$ Die akteurzentrierte Variante orientiert sich weitgehend an den Ergebnissen institutionell eingebetteten Handelns und betont zwar einen Korridor von Autonomie, sieht die Rolle von Institutionen jedoch darin, Handeln zu beschränken oder zu ermöglichen sowie Akteursinteressen und -wahrnehmungen zu beeinflussen. ${ }^{56}$ Streift man die Variante nur kursorisch, so ist festzuhalten, dass es hier um den Einfluss von Regelsystemen auf die Handlungsorientierungen, die Wahrnehmungen oder Präferenzen und Ressourcen von Akteuren respektive ihr Verhalten und ihre Interaktionsformen geht. Insgesamt ist die institutionelle Prägekraft entscheidend, die einen stimulierenden, aber auch restringierenden Kontext bildet und damit für die Verläufe maßgeblich ist. Hinzu kommt, dass die Handelnden Teil einer interagierenden komplexen Akteurskonstellation sind und es daher unwahrscheinlich ist, dass einer allein in der Lage ist, die Ergebnisse zu bestimmen. ${ }^{57}$

Blickt man auf das Bauvorhaben und bezieht das theoretische Vorwissen in die Betrachtung mit ein, wird offenbar, dass eine Projektumkehr schon lange unwahrscheinlich war. Insgesamt wirken die historisch angelegten institutionellen Muster eingeübter Politikprozesse, diverse Fixpunkte im Verlauf und die davon ausgehenden Pfadabhängigkeiten, die die Präferenzen der Akteure strukturieren und insgesamt kaum Spielräume zulassen. Unter anderem die Vorfestlegungen, beginnend im Jahre 1995, die seit 2001 laufenden Planfeststellungsverfahren, die einschlägigen Beschlüsse seit 2006, die daraus resultierenden Verträge und Projektabsprachen sowie die damit einhergehenden Gerichtsentscheidungen und demnach der institutionell restringierende Handlungskontext lassen einen Projektstopp illusorisch erscheinen. Schon deshalb hatte die Schlichtung rein symbolischen Charakter.

Lediglich die Volksabstimmung hätte mit einem eindeutigen Signal noch Wirkungen entfalten können. Im konkreten Fall umschließt der Handlungskontext die Orientierungen der Akteure, deren Wahlhandlungen und Ressourcen äußerst restriktiv. Durch die Interaktion im Zuge der Schlichtung wurde daher auch kein wesentlich neues oder abweichendes Ergebnis produziert.

55 Vgl. Sven Steinmo / Kathleen Thelen / Frank Longstreth (Hrsg.), Structuring Politics. Historical Institutionalism in Comparative Analysis, Cambridge 1992; Kathleen Thelen, How Institutions Evolve. Insights from Comparative-Historical Analysis (MPIFG), Köln 2001; Ellen Immergut, Health Care Policies: Ideas and Institutions in Western Europe, Cambridge 1992; Peter A. Hall I Rosemary C. R. Taylor, Political Science and the Three New Institutionalisms (MPIFG Discussion Paper 6), Köln 1996; Guy P. Peters, Institutional Theory in Political Science. The „New Institutionalism“, London / New York 1999, S. 63 ff.; André Kaiser, Die politische Theorie des NeoInstitutionalismus. James March und Johan Olson, in: André Brodocz / Gary S. Schaal (Hrsg.), Politische Theorien der Gegenwart, Opladen 1999, S. 189 - 211, S. 197.

56 Vgl. Renate Mayntz / Fritz W. Scharpf, Der Ansatz des akteurszentrierten Institutionalismus, in: dies. (Hrsg.), Gesellschaftliche Selbstregelung und politische Steuerung, Frankfurt am Main 1995, S. 39 - 72; Fritz Scharpf, Interaktionsformen. Akteurszentrierter Institutionalismus in der Politikforschung, Opladen 2000; Holger Schulze, Neo-Institutionalismus. Ein analytisches Instrument zur Erklärung gesellschaftlicher Transformationsprozesse, in: Klaus Segbers (Hrsg.), Osteuropa-Institut der Freien Universität Berlin, Arbeitspapiere des Bereichs Politik und Gesellschaft (4), Berlin 1997, S. 5; Arthur Benz, Der moderne Staat. Grundlagen einer politologischen Analyse, München / Wien 2001, S. 75 f.; Elinor Ostrom, Doing Institutional Analysis. Digging Deeper than Markets and Hierarchies, in: Claude Ménard / Mary M. Shirley (Hrsg.), Handbook of New Institutional Economics, Dodrecht 2005, S. 819 - 848.

57 Vgl. André Kaiser, a.a.O. (Fn. 55), S. 191. 


\subsection{Lerntheoretische Chancen}

Fragt man überdies, wie solche Vorhaben im Politikprozess künftig besser gestaltet werden können, so kommt man fast zwangsläufig zu lerntheoretischen Prämissen. ${ }^{58}$ Wandel - und damit auch Veränderungen hin zu anderen demokratischen Formen, wie auch immer diese gestaltet sein mögen - wird vielfach durch Lernmomente ausgelöst und unterstützt. Diesen liegen qualitative Veränderungen des kollektiven Wirklichkeits- und Beziehungsgefüges und damit eine Neuordnung von Strukturmomenten zugrunde. ${ }^{59}$

Eine neue soziale Praxis wird erlernt, also erfunden und implizit oder explizit vereinbart, wenn sie sich als besser, angemessener oder sinnvoller erweist. Als Erklärungsangebot für Lernimpulse gelten demnach Devianzen vom üblichen Ablauf einer Interaktion oder ihre Störungen. ${ }^{60}$ Damit ist allerdings auch die Frage verknüpft, warum Systeme nicht lernen. Nicht-Lernen und Widerstand gegen Wandel (hin zu „mehr“ direkter Demokratie) entstehen, weil Systeme Widersprüche entweder nicht erkennen, als irrelevant abtun und umgehen oder die Situation umdefinieren, so dass sie an ihren tradierten Interpretationsmustern festhalten können. ${ }^{61}$ Solche Mechanismen sind im Sinne der Systemlogik rational, denn das System reproduziert den vertrauten, eingespielten und Sicherheit gewährleistenden Modus, in dem sich die Akteure eingerichtet und bewährt haben. Wandlungsprozesse setzen daher einen Fokus auf die systemische Reproduktionsneigung und die Erkenntnis voraus, dass viele Veränderungen nur als Lernprozess zu gestalten sind, indem sich die Konstrukteure der sozialen Welt ihrer eigenen (Re-)Konstruktionsleistung und damit der komplexen sowie dynamisch-rekursiven Zusammenhänge zwischen ihren Handlungen und den Strukturen bewusst werden. ${ }^{62}$

Wird damit ein Wandel illusorisch? Keinesfalls, denn aufgrund zahlreicher Ereignisse, nicht zuletzt wegen der aktuellen Vorkommnisse um Stuttgart 21, ist die Diskussion bereits in vollem Gange - zumindest was eine systemische Anpassung anbelangt, und jeder Wandel entspringt üblicherweise einer Interaktion. Lerntheorien leisten hierzu ihren Beitrag, weil sie im Gegensatz zu neoinstitutionalistischen Theorien noch mehr den Blick auf die Akteure richten. Projekte dürften daher nur erfolgreich durchgesetzt werden können, wenn sie von kontinuierlichen, inkrementellen Maßnahmen begleitet werden - Maßnahmen, die von überwiegender Akzeptanz gekennzeichnet sind, von einer ausgeprägten Informationsund (zumindest gefühlten) Partizipationspolitik. Wichtig scheint in diesem Kontext, sämtliche Akteursgruppen bereits frühzeitig und aktiv in vorgesehene Ziele einzubeziehen und sie bei deren Erreichung gestalterisch mitwirken zu lassen. ${ }^{63}$

58 Vgl. Peter A. Hall, Policy Paradigms, Social Learning, and the State, in: Comparative Politics, 25. Jg. (1993), H. 3, S. 275 - 296; Nils C. Bandelow, Policy Lernen und politische Veränderungen, in: Klaus Schubert / ders. (Hrsg.), Lehrbuch der Politikfeldanalyse, München 2003, S. 289 - 330.

$59 \mathrm{Vgl}$. Erik Nagel, Verwaltung anders denken. Verwaltungsorganisation, Staatsaufgaben und Öffentlicher Dienst, Baden-Baden 2001, S. 56 f.

60 Vgl. Christof Baitsch, Was bewegt Organisationen? Selbstorganisation aus psychologischer Perspektive, Frankfurt am Main 1993, S. 33; Georg Schreyögg / Christian Noss, Von der Episode zum fortwährenden Prozess. Wege jenseits der Gleichgewichtslogik im Organisatorischen Wandel, in: Georg Schreyögg / Peter Conrad (Hrsg.), Organisatorischer Wandel und Transformation, Wiesbaden 2000, S. $33-62$.

61 Vgl. Christof Baitsch, a.a.O. (Fn. 60), S. $35 \mathrm{ff}$.

62 Vgl. Erik Nagel, a.a.O. (Fn. 59), S. $61 \mathrm{ff}$.

63 Vgl. Werner R. Müller / Martin Hurter, Führung als Schlüssel zur organisationalen Lernfähigkeit, in: Georg Schreyögg / Jörg Sydow (Hrsg.), Führung - neu gesehen, Berlin 1999, S. 1 - 54. 
Sicherlich gibt es kein soziales Handeln ohne Machtbeziehungen. Ohne Macht ist kein Eingriff in bestehende Verhältnisse möglich. Man kann sich im Grunde nur Ziele setzen, für die man genügend Handlungsfähigkeiten mobilisieren kann. Sozialer Wandel ist dabei jedoch nicht zu begreifen als die Durchsetzung eines von wenigen Akteuren festgelegten Modells, sondern nur als kollektiver Prozess, in dessen Verlauf die Mitglieder einer Gesamtheit neue Vorgehensweisen für die soziale Zusammenarbeit und den Konflikt, also eine neue soziale Praxis, erlernen beziehungsweise festlegen und sich die dafür nötigen Fähigkeiten aneignen. Die einzige Alternative zu „autoritären Formen von oben“ liegt in der Ausweitung und progressiven Generalisierung des sozialen Experimentierens, das heißt des kollektiven und institutionellen Lernens, respektive in der Herstellung der Bedingungen, die eine solche Ausweitung ermöglichen. Es geht nicht darum, sich zu einem neuen Modell zu entschließen, sondern darum, einen Wandlungsprozess einzuleiten, der Aktionen, Reaktionen, Verhandlungen und Zusammenarbeit voraussetzt und beinhaltet. Es handelt sich dabei um ein Vorhaben, das nicht den Willen einer Gruppe, sondern die Fähigkeit verschiedener an einem komplexen System beteiligter Gruppen repräsentiert, ihre für die gemeinsame Tätigkeit notwendige Zusammenarbeit anders zu gestalten. Eine Veränderung kann somit nicht einfach aus der Ersetzung eines Zustandes durch ein neuen erfolgen. Die Bedingungen für den Erfolg liegen vielmehr in kollektiven Prozessen, wodurch die Ressourcen und Fähigkeiten der Beteiligten für den Aufbau des Neuen mobilisiert oder sogar erst geschaffen werden, deren freie, nicht erzwungene Anwendung es dem System als Ganzem ermöglicht, sich wie eine menschliche Gesamtheit umzuorientieren. Um in der Lage zu sein, ein neues (kollektives) Modell zu erarbeiten oder festzulegen, muss man mit Interessen, Machtverhältnissen, affektiven Schutzmechanismen und intellektuellen Modellen brechen. ${ }^{64}$

Hinweise, wie Vorhaben wie Stuttgart 21 künftig reibungsloser ablaufen könnten, liefert die Wissenschaft demnach also. Die Fehlerquellen liegen vielmehr in der praktischen Umsetzung, wobei grundsätzlich schon lange klar ist, dass alle Veränderungen - und solche Großprojekte erst recht - automatisch Widerstände hervorrufen. Wie alltäglich ein solcher Vorgang ist und wie illusorisch andere Vorstellungen, beobachtete schon Niccoló Machiavelli: „Stets gilt es zu bedenken, dass nichts schwieriger durchzuführen ist, nichts von zweifelhafteren Erfolgsaussichten begleitet und nichts gefährlicher zu handhaben ist als eine Neuordnung der Dinge." 65 Veränderungen eröffnen eine Unsicherheitszone, die es zu überbrücken gilt. ${ }^{66}$ Dies kann auf unterschiedliche Weise geschehen. Bei Großprojekten dürfte ein hoher Informations- und Partizipationsgrad allerdings unabdinglich sein und damit quasi ein Mehr an direkter Demokratie.

64 Vgl. Michel Crozier / Erhard Friedberg, Macht und Organisation. Die Zwänge kollektiven Handelns, Königstein im Taunus 1979, S. 18 - 20, S. 246 - 248; Jörg Bogumil / Leo Kißler, Verwaltungsmodernisierung als Machtspiel. Zu den heimlichen Logiken kommunaler Modernisierungsprozesse, in: Dietrich Budäus / Peter Conrad / Georg Schreyögg (Hrsg.), Managementforschung, Berlin / New York 1998, S. 123 - 149, S. 139.

65 Zitiert in Rudolf Fisch, Widerstände gegen Veränderungen in Behörden - sozialpsychologische Perspektiven, in: Klaus König (Hrsg.), Verwaltung und Verwaltungsforschung - deutsche Verwaltung an der Wende zum 21. Jahrhundert, Forschungsinstitut für öffentliche Verwaltung bei der Deutschen Hochschule für Verwaltungswissenschaften, Speyerer Forschungsberichte 211, Speyer 2000, S. $117-166$.

66 Vgl. Markus Reiners, Gescheiterte Übersteuerung. Verflechtungen, Netzwerke und Mikropolitik bei der Neuen Steuerung, Wiesbaden 2012, S. 13 - 28, S. 19. 


\section{Mehr Beteiligung - aber um welchen Preis?}

Die Vorgänge um Stuttgart 21 haben gelehrt, den demokratisch-emanzipatorischen Diskurs fortzuführen und kritisch zu beleuchten, ob es sinnvoll ist, nachdrücklicher für den Ausbau von direktdemokratischen Instrumenten zu werben, denn heute ist ein höheres $\mathrm{Ma}$ an demokratischer, medialer Öffentlichkeit und ein schnellerer Zugang zu mannigfaltigen sowie äußerst komplexen und nur noch schwer zu verarbeitenden Informationen zu diagnostizieren. Allerdings bleibt zu bedenken, dass der Preis für eine auf diese Weise erreichte stärkere legitimatorische Abstützung von Entscheidungen eine Abnahme der Steuerungs- und Problemlösungsfähigkeit ist. Plebiszite werfen also eher neue Probleme auf und stehen Innovationen entgegen.

Formale Referenden allein dürften nicht ausreichen, wenngleich der einmalige Vorgang die kluge Amalgamierung des Referendums aufzeigt, nach ausgiebigen Parlamentsdebatten die Entscheidung zur Abstimmung zu stellen und dies in einer Form, die den Großteil der Bürger nicht kompromissunfähig erscheinen lässt. Gleichwohl könnte man auch andere partizipative Möglichkeiten institutionalisieren: zum Beispiel ein höheres Maß an Informationspolitik, diverse Mediationsverfahren im Vorfeld von Großprojekten, sozusagen als „legitimatorische Absicherung “ formaler Folgeprozesse, und bei gewichtigen Entscheidungen tatsächlich - parallel und rechtzeitig - die Einleitung einer Volksabstimmung. Es scheint so, dass sich durch Stuttgart 21 ein Gelegenheitsfenster eröffnet hat, bei solchen Projekten künftig das Volk direkt einzubeziehen - oder auch nicht: Die hohe Beteiligung würde bei weiteren Volksabstimmungen vermutlich absinken. Überdies: wo liegt die Grenze für ein Großprojekt, wo für eine gewichtige Entscheidung? Sind dann nicht Endlosdiskussionen vorprogrammiert, wo exakt solche Grenzen zu ziehen sind? Geraten gerade solche Fragen in den Mittelpunkt und wird daher künftig noch weniger über Politikinhalte debattiert oder noch exakter: Wäre das der Weg von der repräsentativen Demokratie in eine „Diskussionsdemokratie"?

Gegebenenfalls könnten beispielsweise schlichte Befragungen der Öffentlichkeit im Vorfeld größerer Projekte weiterhelfen, demnach eine Ergänzung des parlamentarischen Systems durch Beteiligungsmöglichkeiten stattfinden, solange es noch Alternativen zu diskutieren gibt. Zweifelhaft bleibt aber auch hier: Welche Gruppen bringen sich bei solchen Optionen ein, und ist dadurch eine breitere Repräsentation garantiert? Sind solche Umfragen nicht von aktuellen Stimmungslagen geprägt, die laufend Veränderungen unterworfen sind? Welche Interessen stehen hinter den Teilnehmern oder gar den Initiatoren solcher Instrumentarien? Kann so etwas die Sachdiskussion tatsächlich unterstützen? Hier bleibt noch viel Raum für die wissenschaftliche Forschung und für politische Diskussionen. 